\title{
Knockdown of long non-coding RNA PVT1 induces apoptosis and cell cycle arrest in clear cell renal cell carcinoma through the epidermal growth factor receptor pathway
}

\author{
WEICONG LI ${ }^{1,2^{*}}$, ZAOSONG ZHENG $^{1 *}$, HAICHENG CHEN $^{1}$, YUHONG CAI $^{1}$ and WENLIAN XIE ${ }^{1}$ \\ ${ }^{1}$ Department of Urology; ${ }^{2}$ Guangdong Provincial Key Laboratory of Malignant Tumor Epigenetics and Gene Regulation, \\ Sun Yat-sen Memorial Hospital, Sun Yat-sen University, Guangzhou, Guangdong 510120, P.R. China
}

Received August 21, 2017; Accepted February 15, 2018

DOI: $10.3892 / \mathrm{ol} .2018 .8315$

\begin{abstract}
Previous years have witnessed the importance of long non-coding RNAs (lncRNAs) in cancer research. The lncRNA Pvt1 oncogene (non-protein coding) (PVT1) was revealed to be upregulated in various cancer types. The aim of the present study was to investigate the function of PVT1 in clear cell renal cell carcinoma (ccRCC). The expression of PVT1 in ccRCC was analyzed using reverse transcription-quantitative polymerase chain reaction, and it was revealed that PVT1 expression was upregulated in ccRCC tissues compared with that in normal adjacent tissues. Next, PVT1 expression from The Cancer Genome Atlas datasets was validated, and it was also revealed that the high expression of PVT1 was associated with advanced disease stage and a poor prognosis. Furthermore, the knockdown of PVT1 induced apoptosis by increasing the expression of poly ADP ribose polymerase and $\mathrm{Bcl}-2$-associated $\mathrm{X}$ protein, and promoted cell cycle arrest at the G1 phase by decreasing the expression of cyclin D1. Study of the mechanism involved indicated that PVT1 promoted the progression of ccRCC partly through activation of the epidermal growth factor receptor pathway. Altogether, the results of the present study suggested that PVT1 serves oncogenic functions and may be a biomarker and therapeutic target in ccRCC.
\end{abstract}

\section{Introduction}

Renal cancer types are the most aggressive common malignancies of all urological cancer types, amongst which

Correspondence to: Dr Wenlian Xie, Department of Urology, Sun Yat-sen Memorial Hospital, Sun Yat-sen University, 107 Yan Jiang West Road, Guangzhou, Guangdong 510120, P.R. China E-mail:wenlianxie@sina.com

*Contributed equally

Key words: long non-coding RNA, clear cell renal cell carcinoma, Pvtl oncogene (non-protein coding), apoptosis, cell cycle arrest, epidermal growth factor receptor pathway clear cell renal cell carcinoma (ccRCC) is the the most prevalent (1). The incidence of ccRCC has increased during past 3 decades, particularly in high income countries (2). To date, several pathological and clinical features have been used to predict patient prognosis, including tumor size, pathological stage, tumor stage, performance status, localized symptoms and cachexia (3). However, few molecular biomarkers exist that may predict patient prognosis and serve as therapeutic targets, although numerous molecular biomarkers have been investigated in ccRCC (4-5). Previous studies have highlighted the mutations of BRCA1 associated protein-1 and SET domain containing 2, which are inversely correlated with the outcome of patients with ccRCC (6-8). Unfortunately, their use is so limited in clinical practice that further studies are required in order to identify the mechanism of ccRCC.

Long non-coding RNAs (IncRNAs) are a notable subtype of non-coding RNAs that are $>200 \mathrm{nt}$ in length, but are unable to translate proteins (9). Previous years have witnessed the importance of lncRNAs in cancer research and revealed their notable impact on cancer cell biology (10). lncRNAs serve crucial regulatory functions in the biological processes of cancer, including chromatin modification, transcription, post-transcriptional processing and translation. Emerging evidence has indicated that IncRNAs may be potential biomarkers and therapeutic targets $(11,12)$. IncRNA Pvt1 oncogene (non-protein coding) (PVT1), located at 8q24.21, was revealed to be upregulated and regulated in the biological processes of various cancer types, including gastric, lung, pancreatic and breast cancer (13-15). However, the potential functions of PVT1 and its underlying mechanism in ccRCC remain unclear.

The present study aimed to investigate the potential biological functions of PVT1 in ccRCC and determine whether PVT1 may serve as a reliable prognostic marker for the disease. PVT1 expression in ccRCC tissues was investigated and validated using The Cancer Genome Atlas (TCGA) database. The association between PVT1 expression and clinical parameters and prognosis of ccRCC patients was also assessed. Furthermore, the function and underlying molecular mechanism of PVT1 in apoptosis and the cell cycle of ccRCC cells was evaluated. 


\section{Materials and methods}

Patient and tissue samples. A total of 40 paired ccRCC tissues and normal adjacent tissues, which were obtained from patients (30 males, 10 females; age range 31-66 years; mean age, 51 years) who underwent radical nephrectomy at Sun Yat-sen Memorial Hospital, Sun Yat-sen University (Guangzhou, China), were collected. Patients who had received any chemotherapy or targeted treatments were excluded. The pathological features of the samples were confirmed by pathologists at Sun Yat-sen Memorial Hospital. All tissues were stored at $-80^{\circ} \mathrm{C}$ immediately after surgery for reverse transcription-quantitative polymerase chain reaction (RT-qPCR) analysis. All tissue samples were collected with written informed consent from all patients and the study was ethically approved by the Ethic Review Committee of Sun Yat-sen Memorial Hospital.

ccRCC data from TCGA database. The clinical and pathological information of 517 patients with ccRCC were downloaded from TCGA database (http://cancergenome. nih.gov/) (16). TCGA gene expression profile was measured using the Illumina HiSeq 2000 RNA Sequencing platform (Illumina, Inc., San Diego, CA, USA). RNA-Seq by Expectation-Maximization (RSEM software v1.3.0; provided by the University of Wisconsin, Madison, WI, USA) normalized count data was used in this study. Copy number alterations were estimated by Genomics Identification of Significant Targets in Cancer 2.0 (GISTIC 2.0; provided by the Broad Institute of MIT and Harvard, Cambridge, MA, USA) (17), which defined the copy-number alteration of each gene as $-2,-1,0,1$ or 2 , representing homozygous deletion, heterozygous deletion, diploid normal copy number, low-level amplification (gain) and high-level amplification, respectively. For analysis, the amplification and gain groups were combined and the homozygous and heterozygous groups were also combined. The RNA sequences of 448 ccRCC tissues were downloaded from the Atlas of Noncoding RNAs in Cancer (TANRIC) database (http://ibl.mdanderson.org/tanric/_design/basic/index.html). The patients were divided into high and low PVT1 expression groups. The median value of PVT1 expression (0.803) was used as the cut-off value.

Cell culture. RCC cell lines, including 786-O, Caki-1, ACHN and 769-P, and the human normal kidney cell line HK-2 were obtained from the American Tissue Culture Collection (ATCC; Manassas, VA, USA). 786-O and 769-P cells were cultured in RPMI-1640 medium supplemented with $10 \%$ heat-inactivated fetal bovine serum (FBS; Hyclone; GE Healthcare Life Sciences, Logan, UT, USA). Caki-1 cells were cultured in ATCC-formulated McCoy's 5a Medium Modified medium (Gibco; Thermo Fisher Scientific, Inc., Waltham, MA, USA) supplemented with $10 \%$ heat-inactivated FBS. ACHN cells were cultured in Dulbecco's modified eagle's medium (Gibco; Thermo Fisher Scientific, Inc.) supplemented with $10 \%$ heat-inactivated FBS. HK-2 cells were cultured in keratinocyte-SFM (Gibco; Thermo Fisher Scientific, Inc.) supplemented with $10 \%$ heat-inactivated FBS. All cells were incubated at $37^{\circ} \mathrm{C}$ with $5 \% \mathrm{CO}_{2}$ once seeded. The medium was replaced every 2 days.
Small interfering RNA (siRNA) transfection and RNA extraction. Using Lipofectamine ${ }^{\circledR}$ RNAiMAX (Invitrogen; Thermo Fisher Scientific, Inc.), ccRCC cells (786-O and Caki-1) were transfected with siRNA at $37^{\circ} \mathrm{C}$ for 2 days according to the manufacturer's protocol. A total of $1 \times 10^{5} \mathrm{ccRCC}$ cells and $5 \mu \mathrm{l}$ siRNA per well was used in each transfection in a 6-well plate. siRNAs were obtained from Shanghai GenePharma Co., Ltd. (Shanghai, China). The siRNA sequences were as follows: Si-PVT1a, 5'-CAGCCAUCAUGAUGGUACUTT-3' and si-PVT1b, 5'-GGCACAUUUCAGGAUACUATT-3'. Negative control siRNA for PVT1, 5'-UUCUCCGAACGUGUCACG UTT-3'. Total RNA was extracted at 2 days post-transfection using TRIzol reagent (Thermo Fisher Scientific, Inc.) according to the manufacturer's protocol.

Cell proliferation assay. ccRCC Cells (786-O and Caki-1) transfected with si-PVT1 and negative control were harvested at $24 \mathrm{~h}$ post-transfection and $1 \times 10^{3}$ cells were cultured on 96 -well plates. Cells were incubated with CellTiter $96^{\circledR}$ Aqueous One Solution Reagent kit (MTS; Promega Corporation, Madison, WI, USA) at $37^{\circ} \mathrm{C}$ for $3 \mathrm{~h}$ and assessed every $24 \mathrm{~h}$ for 5 days according to the manufacturer's protocol. Absorbance was measured with the multifunctional microplate reader SpectraMax M5 (Molecular Devices, LLC, Sunnyvale, CA, USA) at $490 \mathrm{~nm}$.

Flow cytometry analysis. ccRCC Cells (786-O and Caki-1) transfected with si-PVT1 and negative control were harvested at $48 \mathrm{~h}$ post-transfection. A total of $2 \times 10^{5}$ cells for each analysis of apoptosis were stained at room temperature for $15 \mathrm{~min}$ with fluorescein isothiocyanate-Annexin $\mathrm{V}$ and propidium iodide (Nanjing KeyGen Biotech Co., Ltd., Nanjing, China) and analyzed using the FACSVerse flow cytometer (BD Biosciences, Franklin Lakes, NJ, USA). Early apoptotic cells were assessed and compared. A total of $1 \times 10^{5}$ cells for each cell cycle analysis were fixed in $75 \%$ ethanol at $4^{\circ} \mathrm{C}$ overnight and then stained with propidium iodide at room temperature for 15 min using a cell cycle detection kit (Nanjing KeyGen Biotech, Co., Ltd.) according to the manufacturer's protocol. The percentages of cells in the $G_{0} / G_{1}, S$ and $G_{2} / M$ phases were assessed within $4 \mathrm{~h}$. The acquired data were analyzed using FlowJo 10.0 (FlowJo LLC, Ashland, OR, USA).

$R T$ - $q P C R$. Total RNA of ccRCC cells (786-O and Caki-1) was extracted at 2 days post-transfection using TRIzol reagent (Thermo Fisher Scientific, Inc.) according to the manufacturer's protocol. Total RNA was reverse transcribed into cDNA using the PrimeScript ${ }^{\mathrm{TM}}$ RT Master mix (Takara Biotechnology Co., Ltd., Dalian, China). RT-qPCR was performed using SYBR-Green PCR Master mix (Roche Diagnostics, Basel, Switzerland) with the Roche LightCycler 480 (Roche Diagnostics). GAPDH was used as the internal control. The RT-qPCR reaction included a preincubation step $\left(95^{\circ} \mathrm{C}\right.$ for $300 \mathrm{sec})$, and 40 cycles of amplification step $\left(95^{\circ} \mathrm{C}\right.$ for $15 \mathrm{sec}$, $56^{\circ} \mathrm{C}$ for $15 \mathrm{sec}$ and $72^{\circ} \mathrm{C}$ for $\left.15 \mathrm{sec}\right)$, a melting step $\left(95^{\circ} \mathrm{C}\right.$ for $10 \mathrm{sec}, 65^{\circ} \mathrm{C}$ for $60 \mathrm{sec}, 97^{\circ} \mathrm{C}$ for $\left.1 \mathrm{sec}\right)$ and a cooling step $\left(37^{\circ} \mathrm{C}\right.$ for $30 \mathrm{sec})$. The $2^{-\Delta \Delta \mathrm{Cq}}$ method was used to compare expression (18). The sequences of the primers used are as follows: GAPDH sense, 5'-GAGCCAAAAGGGTCATCATCTC-3' and antisense, 5'-GGTCATGAGTCCTTCCACGATAC-3'; PVT1 


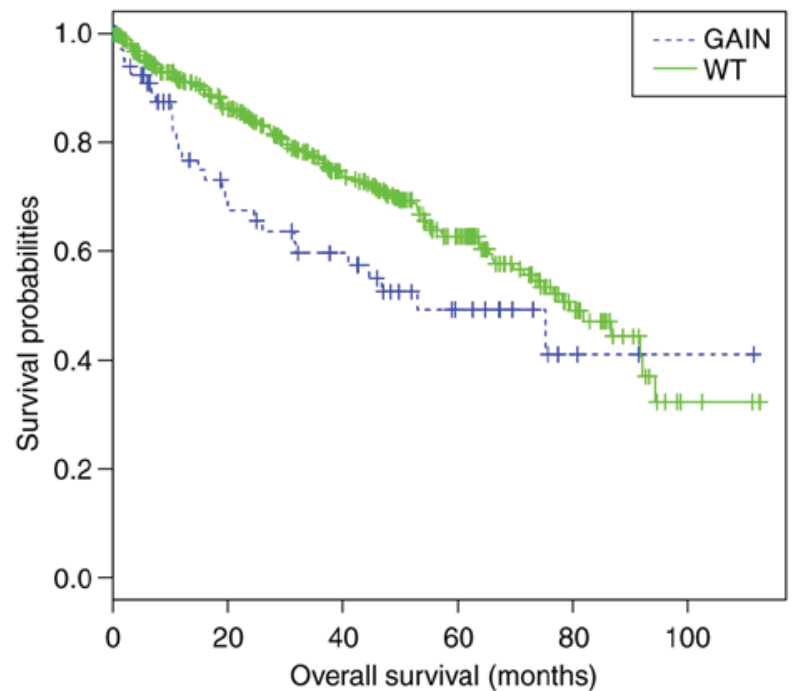

Figure 1. Survival with regard to PVT1 gain. Patients with PVT1 gain experienced a significantly shorter overall survival time compared with those without PVT1 gain. $\mathrm{P}<0.01$ vs. WT. PVT1, Pvt1 oncogene (non-protein coding); WT, wild-type.

sense, 5'-CATCCGGCGCTCAGCT-3' and antisense, 5'-TCA TGATGGCTGTATGTGCCA-3'; EGFR sense, 5'-CGGGAC ATAGTCAGCAGTG-3' and antisense, 5'-GCTGGGCACAGA TGATTTTG-3'; MYC proto-oncogene, bHLH transcription factor (MYC) sense, 5'-CTTCTCTCCGTCCTCGGATTCT-3' and antisense, 5'-GAAGGTGATCCAG-3'; protein kinase B (AKT) sense, 5'-ACGGGCACATTAAGATCACA-3' and antisense, 5'-TGCCGCAAAAGGTCTTCATG-3'; cyclin D1 sense, 5'-GAAGGTGATCCAG-3' and antisense, 5'-GGCGGA TTGGAAATGAACTT-3'; and p21 sense, 5'-CGATGCCAA CCTCCTCAACGA-3' and anti-sense, 5'-CATCCGGCGCTC AGCT-3'.

Western blot analysis. A total of $1 \times 10^{6}$ ccRCC cells (786-O and Caki-1) were harvested at $48 \mathrm{~h}$ post-transfection and lysed with radioimmunoprecipitation assay protein extraction reagent (Beyotime Institute of Biotechnology, Haimen, China) supplemented with a protease inhibitor cocktail (Roche Diagnostics). The total protein concentration was measured using a Bicinchoninic Acid Protein Assay kit (Thermo Fisher Scientific, Inc., Waltham, MA, USA) with Multiskan MK3 (Thermo Fisher Scientific, Inc., Waltham, MA, USA). A total of $50 \mu \mathrm{g}$ protein was added to each panel of 10 (for cyclin D1, Bax, p21, Myc, AKT and p-AKT) or 7.5\% (for PARP and EGFR) sodium dodecyl sulfate-polyacrylamide gel according to the molecular weight of the targeted protein. Protein was transferred onto $0.22-\mu \mathrm{m}$ nitrocellulose membranes (EMD Millipore, Billerica, MA, USA) following electrophoresis. The membranes were blocked with $5 \%$ skimmed milk at room temperature for $1 \mathrm{~h}$, followed by incubation with the primary antibodies at $4^{\circ} \mathrm{C}$ overnight. The membranes were then incubated with corresponding horseradish peroxidase-conjugated goat anti-rabbit secondary antibody (sc-2004; 1:5,000; Santa Cruz Biotechnology, Inc., Dallas, TX, USA) at room temperature for $1 \mathrm{~h}$. Primary antibodies (1:1,000 dilution) were against PARP (cat. no. 9532), EGFR (cat. no. 2085), MYC (cat. no. 13987), p21 (cat. no. 2947), cyclin D1 (cat. no. 2922), Bax (cat. no. 5023), AKT (cat. no. 4685) and p-AKT (cat. no. 4060; Cell Signaling Technology, Inc., Danvers, MA, USA). GAPDH was used as the reference gene for EGFR, AKT, MYC and $\mathrm{p}$-AKT, and $\beta$-tubulin was used as the reference gene for PARP, cyclin D1, Bax and p21.

Statistical analysis. $\chi^{2}$ or Kruskal-Wallis tests were used to analyze the association between the expression of PVT1 and the clinicopathological parameters. The Kaplan-Meier method and log-rank test were used for survival analyses. $\mathrm{P}<0.05$ was considered to indicate a statistically significant difference. Statistical analyses were performed using SPSS software 17.0 (SPSS Inc., Chicago, IL, USA). All these experiments were performed three times.

\section{Results}

PVT1 copy number variant in ccRCC affects overall survival. Of the 517 ccRCC cases selected from TCGA, 70 cases were identified with PVT1 gain/amplification. Next, the association between PVT1 copy number and patient prognosis was analyzed. Kaplan-Meier analysis revealed that patients with PVT1 gain experienced significantly worse overall survival compared with patients without PVT1 gain (log-rank, P<0.01; Fig. 1).

PVT1 is upregulated in ccRCC tissues. In order to investigate the role of PVT1 in ccRCC, the expression of PVT1 in 40 paired ccRCC tissues and normal adjacent tissues was examined using RT-qPCR. A fold change of $>1.5$ was designated as upregulated, and the results revealed that PVT1 was upregulated in 33 (82.5\%) of ccRCC tissue cases compared with normal adjacent tissues (Fig. 2A). As presented in Fig. 2B, the mean expression of PVT1 was significantly higher in tumor tissues compared with that in adjacent normal tissues $(\mathrm{P}<0.001)$.

To further validate the expression of PVT1 in ccRCC, PVT1 RNA-seq expression values from the TCGA database were analyzed. It was identified that PVT1 was significantly upregulated in ccRCC tissues $(\mathrm{n}=448)$ compared with that in normal kidney tissues ( $\mathrm{n}=67)$ (Fig. 2C).

PVT1 expression is associated with the poor prognosis of patients with $c c R C C$. Subsequently, the association between PVT1 expression and clinical characteristics was analyzed. The results revealed that PVT1 expression was significantly associated with $\mathrm{T}$ stage $(\mathrm{P}<0.001)$, $\mathrm{M}$ stage $(\mathrm{P}=0.024)$, AJCC stage $(\mathrm{P}<0.001)$ and patient age $(\mathrm{P}=0.018)$ (Table I). No significant association was identified between PVT1 expression and patient sex, $\mathrm{N}$ stage and tumor grade. Next, the association between PVT1 expression and the prognosis of patients with ccRCC was assessed. The patients with ccRCC were separated into the high PVT1 expression group $(n=224)$ and the low PVT1 expression group $(\mathrm{n}=224)$ according to PVT1 expression, and Kaplan-Meier analysis was performed. The result revealed that the overall survival time was significantly shorter in the high PVT1 expression group compared with that in the low PVT1 expression group (log-rank, P<0.01; Fig. 2D).

Knockdown of PVT1 significantly inhibits the proliferation of ccRCC cells. In order to investigate the potential biological 
functions of PVT1 in ccRCC, the expression of PVT1 in ccRCC cell lines (786-O, Caki-1, ACHN and 769-P) and one normal kidney cell line (HK-2) was examined using RT-qPCR. The results revealed that PVT1 expression was significantly higher in ccRCC cells compared with that in HK-2 cells (Fig. 3A). 786-O and Caki-1 cells were then transfected with si-PVT1s or si-NC using Lipofectamine RNAiMAX Transfection reagent, and the transfection efficiency was tested using RT-qPCR. The results revealed that the expression of PVT1 was significantly reduced in cells transfected with si-PVT1a and si-PVT1b compared with that in cells transfected with si-NC (Fig. 3B). Next, an MTS assay was performed to examine the effect of PVT1 on the proliferation of the ccRCC cells. The MTS assay revealed that the knockdown of PVT1 significantly inhibited the proliferation of 786-O and Caki- 1 cells in a time-dependent manner compared with cells in the NC group (Fig. 3C).

Knockdown of PVTl significantly induces apoptosis and promotes cell cycle arrest in ccRCC cells. To further investigate the effect of PVT1 on the growth of ccRCC cells, cell apoptosis analysis and cell cycle analysis were performed using flow cytometry. Cell apoptosis analysis indicated that the knockdown of PVT1 significantly increased the apoptosis rate in 786-O and Caki-1 cells, most notably in 786-O si-PVT1a group compared with the NC group $(\mathrm{P}<0.05$; Fig. 3D). Cell cycle analysis revealed that the knockdown of PVT1 also significantly promoted cell cycle arrest at the G0/G1 phase compared with the normal control $(\mathrm{P}<0.05$; Fig. $3 \mathrm{E})$. No significant difference in G1 phase was found between si-PVT1a and si-PVT1b.

Next, western blotting was performed to support the results of flow cytometry. The results demonstrated that the knockdown of PVT1 increased the level of Bax and the cleavage of PARP, which serve notable functions in the activation of cell apoptosis. Meanwhile, the knockdown of PVT1 decreased the level of cyclin D1, a major mediator of the cell cycle, and increased the level of p21 (Fig. 4).

PVT1 regulates the activation of the EGFR pathway in $c c R C C$ cells. To investigate the underlying molecular mechanisms of PVT1 function, a potential pathway involved in cell proliferation was investigated. As EGFR pathways serve a notable role in the cell proliferation, apoptosis and cell cycle of ccRCC $(19,20)$, the mRNA and protein expression levels of EGFR following the transfection of si-PVT1a and $\mathrm{b}$ and si-NC were investigated. The results demonstrated that the mRNA expression level of EGFR was significantly downregulated following the knockdown of PVT1 compared with that of the NC (Fig. 5A), and the protein expression was also notably downregulated (Fig. 5B). Furthermore, AKT and MYC, the downstream proteins of EGFR, were detected at the mRNA and protein level following the knockdown of PVT1. It was revealed that the mRNA expression levels of AKT and MYC were significantly downregulated in si-PVT1 transfected groups compared with that in the $\mathrm{NC}$ groups (Fig. 5A), and the protein expression of AKT, phosphorylated (p-)AKT and MYC in cells transfected with si-PTV1 were notably downregulated compared with that in the NC groups (Fig. 5B). The results indicated that PVT1 may potentially regulate cell proliferation, apoptosis and
Table I. Association between Pvt1 oncogene (non-protein coding) expression and clinical characteristics of patients with clear cell renal cell carcinoma.

\begin{tabular}{lrccc}
\hline Characteristics & $\mathrm{n}$ & Expression & F-value & P-value \\
\hline Sex & & & 0.982 & 0.322 \\
Male & 288 & 0.656 & & \\
Female & 160 & 0.759 & & \\
Age, years & & & 5.633 & $0.018^{\mathrm{a}}$ \\
$<60$ & 205 & 0.566 & & \\
$\geq 60$ & 243 & 0.800 & & \\
T stage & & & 17.90 & $<0.001^{\mathrm{a}}$ \\
T1-2 & 276 & 0.530 & & \\
T3-4 & 172 & 0.953 & & \\
M stage & & & 5.102 & $0.024^{\mathrm{a}}$ \\
M0 & 376 & 0.644 & & \\
M1 & 72 & 0.947 & & \\
N stage & & & 2.934 & 0.088 \\
N0 & 218 & 0.594 & & \\
N1 & 16 & 1.084 & & \\
NX & 214 & 0.766 & & \\
Tumor grade & & & 0.017 & 0.895 \\
G1-2 & 208 & 0.706 & & \\
G3-4 & 235 & 0.693 & & \\
GX & 5 & 0.162 & & \\
AJCC stage & & & 14.78 & $<0.001^{\mathrm{a}}$ \\
I & 215 & 0.477 & & \\
II & 46 & 0.626 & & \\
III & 112 & 0.966 & & \\
IV & 75 & 0.942 & & \\
& & & \\
\hline
\end{tabular}

${ }^{\mathrm{a}} \mathrm{P}<0.05$ was considered to indicate a statistically significant difference. AJCC, american joint committee on cancer.

the cell cycle of ccRCC cells through the activation of the EGFR pathway.

\section{Discussion}

Emerging evidence has revealed that lncRNAs are of great importance in cell development and human diseases (21-23). lncRNAs have different expression patterns in different diseases, and their aberrant expression may be involved in the pathophysiology of various diseases (24). Studies have demonstrated that lncRNAs may be involved in the progression of various cancer types, including breast and lung cancer $(13,18)$. To date, only a few lncRNAs have been studied in renal cancer. Zhang et al (25) identified that the overexpression of metastasis associated lung adenocarcinoma transcript 1 (non-protein coding) (MALAT1) may predict the poor prognosis of patients with renal cancer, and Hirata et al (26) demonstrated that MALAT1 promoted tumorigenesis through enhancer of zeste homolog 2. The functions of growth arrest-specific 5 (27), neuroblastoma-associated transcript 1 (28) and HOX transcript 

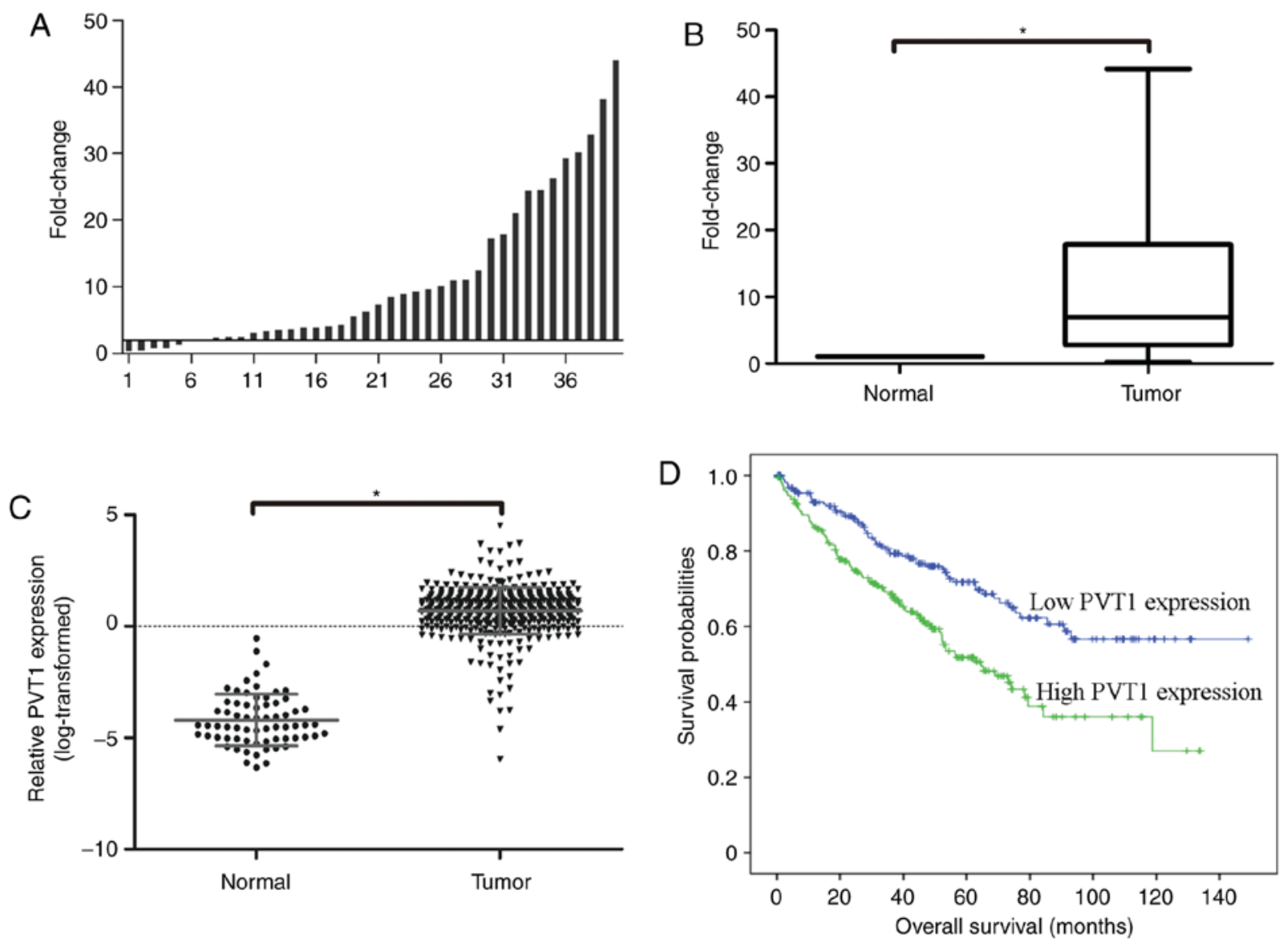

Figure 2. Upregulation of PVT1 in ccRCC. (A) Relative PVT1 expression in 40 pairs of ccRCC and paired adjacent tissues was detected using reverse transcription-quantitative polymerase chain reaction. (B) Mean expression of PVT1 in ccRCC tissues and adjacent normal tissues ( $\mathrm{P}<0.05$ vs. normal) tissues. (C) Expression of PVT1 in ccRCC tissues and normal tissues from The Cancer Genome Atlas ("P<0.05 vs. normal). (D) Patients with high PVT1 expression experienced significantly shorter overall survival times compared with the low PVT1 expression group. Data are presented as the mean \pm standard deviation. PVT1, Pvt1 oncogene (non-protein coding); ccRCC, clear cell renal cell carcinoma.

antisense RNA (29) in the progression of ccRCC were also investigated.

PVT1, an lncRNA mapped at 8q24, was demonstrated to be involved in the tumorigenesis of various cancer types. A previous study suggested that PVT1 expression was significantly higher in colorectal cancer tissues compared with that in normal tissues, and that the knockdown of PVT1 may inhibit cell proliferation (30). Wan et al (13) additionally revealed that PVT1 promoted lung cancer cell proliferation through the regulation of large tumor suppressor kinase 2. Various studies additionally revealed that PVT1 influenced the expression of MYC (14,31). All these reports indicated that PVT1 may be involved in cancer pathophysiology (32). However, to the best of our knowledge, no previous study has reported the expression pattern and biological function of PVT1 in ccRCC.

In the present study, the association between PVT1 expression and ccRCC was investigated. PVT1 was revealed to be significantly upregulated in ccRCC tissues compared with that in adjacent normal tissues $(\mathrm{P}<0.001)$. Furthermore, high PVT1 expression was associated with advanced ccRCC stage. The results demonstrated that PVT1 expression was significantly associated with age $(\mathrm{P}=0.018)$. PVT1 expression in patients $\geq 60$ years old was significantly higher compared with that in patients $<60$ years old. Survival analysis revealed that the overall survival time was significantly shorter in the high PVT1 expression group compared with that in the low PVT1 expression group $(\mathrm{P}<0.01)$. It was hypothesized that the association between PVT1 expression and age affected the survival analysis to a certain extent. Although PVT1 expression is associated with age, PVT1 expression may be an independent prognostic factor for patients with ccRCC. Furthermore, the findings were confirmed by TCGA database, as its cohort size was large enough and the follow-up time was long enough to draw a conclusion without substantial bias, and it demonstrated that the survival time of patients with high PVT1 expression was shorter compared with that of patients with low PVT1 expression. The results revealed that PVT1 expression was significantly associated with $\mathrm{T}$ stage $(\mathrm{P}<0.001)$ and $\mathrm{M}$ stage $(\mathrm{P}=0.024)$, but not with tumor grade. Tumor grading is a measure of cell differentiation and is based on the resemblance of the tumor to the tissue of origin. Tumor grading is distinguished from staging, which is a measure of the extent to which the cancer has spread (33). This finding may be due to the fact that PVT1 serves an important role in the cell cycle and cell proliferation, which are associated with advanced $\mathrm{T}$ stage and $\mathrm{M}$ stages. Additionally, the differentiation of RCC cells was not markedly affected by PVT1, resulting in non-significant differences between PVT1 expression and tumor grade. All these results suggested that PVT1 may promote the tumorigenesis of ccRCC. 

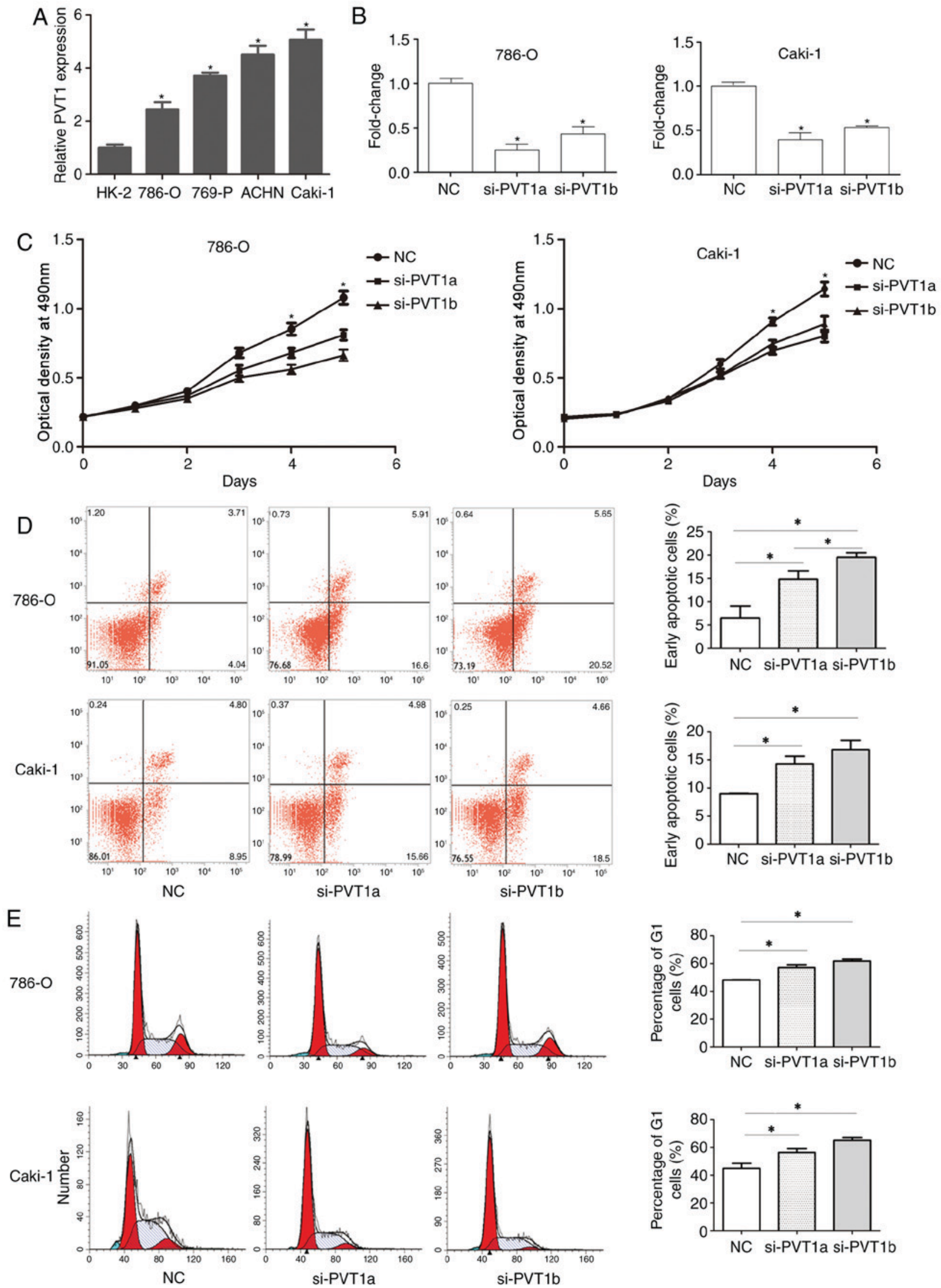

Figure 3. Knockdown of PVT1 inhibits proliferation, induces apoptosis and promotes cell cycle arrest in ccRCC cells. (A) Expression of PVT1 in ccRCC cells and normal kidney cells ("P $<0.05$ vs. HK-2). (B) si-PVT1 significantly decreased the expression of PVT1 in 7860 and Caki-1 cells compared with cells transfected with si-NC ( $\mathrm{P}<0.05$ vs. NC). (C) Knockdown of PVT1 inhibited cell proliferation. Cell proliferation was detected using the MTS method ("P<0.05 vs. NC). (D) Knockdown of PVT1 induced apoptosis. Apoptosis was detected by flow cytometry ("P $<0.05$ vs. NC) ("P $<0.05$ si-PVT1a vs. si-PVT1b in 786-O). (E) Knockdown of PVT1 resulted in cell cycle arrest at the G1 phase. Cell cycle was detected using flow cytometry ("P<0.05 vs. NC). Data are shown as the mean \pm standard deviation. PVT1, Pvt1 oncogene (non-protein coding); ccRCC, clear cell renal cell carcinoma; si-PVT1, small interfering RNA for PVT1; NC, negative control; OD, optical density. 


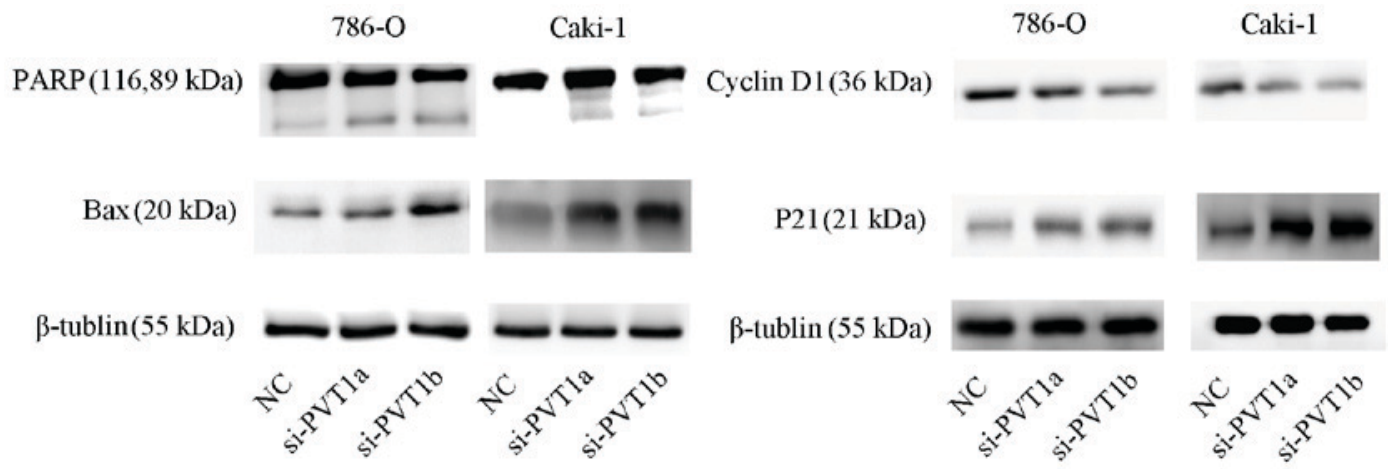

Figure 4. Knockdown of PVT1 increases the expression of cleaved PARP, Bax and P21 and decreased the expression of cyclin D1 compared with the NC groups. $\beta$-tubulin was used as a reference gene. PVT1, Pvt1 oncogene (non-protein coding); si-PVT1, small interfering RNA for PVT1; NC, negative control; PARP, poly ADP ribose polymerase; Bax, Bcl-2-associated X protein; p21, cyclin-dependent kinase inhibitor 1A.
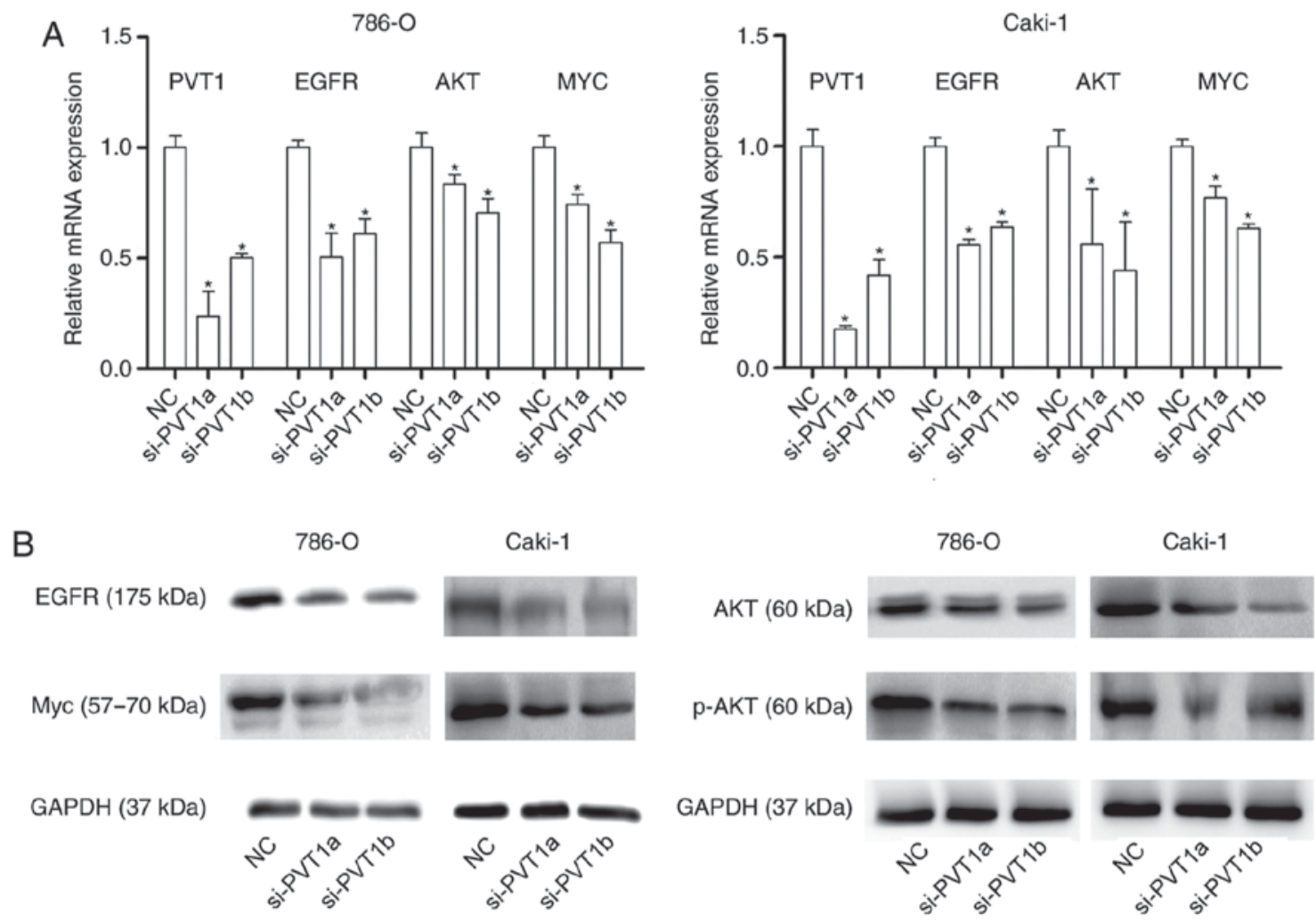

Figure 5. PVT1 regulates the activation of the EGFR pathway in clear cell renal cell carcinoma cells. (A) Reverse transcription-quantitative polymerase chain reaction examined the mRNA expression of EGFR, AKT and MYC following PVT1-knockdown ("P $<0.05$ vs. NC). (B) Western blotting examined the protein expression of EGFR, AKT, p-AKT and MYC following PVT1-knockdown. GADPH was used as the reference gene. PVT1, Pvt1 oncogene (non-protein coding); EGFR, epidermal growth factor receptor; AKT, protein kinase B; MYC, MYC proto-oncogene, BHLH transcription factor; p-, phosphorylated; NC, negative control.

To elucidate how PVT1 affects the biological behavior of ccRCC cells, a series of experiments was designed to evaluate the biological function of PVT1. It was revealed that the knockdown of PVT1 induced cell apoptosis and promoted cell cycle arrest at the G1 phase. The mitochondria-mediated intrinsic pathway and the death receptor-induced extrinsic pathway are the major pathways for the regulation of cell apoptosis $(34,35)$. Bax is a key component for cellular induced apoptosis, which increases the permeability of the mitochondrial membrane and results in the activation of the caspase pathway for apoptosis (36). Western blotting in the present study revealed that the knockdown of PVT1 increased the level of Bax and the cleavage of PARP, a marker of cells undergoing apoptosis (37). Therefore, the results of the present study indicated that the knockdown of PVT1 induced cell apoptosis through the mitochondria-dependent pathway. In addition, the knockdown of PVT1 induced cell cycle arrest at the G1 phase by regulating the level of cyclin D1, which is an important protein for controlling cell transition through the G1 phase into the DNA synthesis S phase (38).

EGFR family members are involved in the mechanisms of cell proliferation and apoptosis, and the cell cycle (39-41). EGFR signaling may regulate the biological behavior of cancer cells through AKT, MAPK and numerous other 
pathways (42). In the present study, it was revealed that silencing PVT1 decreased the expression of EGFR and its downstream protein AKT, and p-AKT. Research has also indicated that MYC expression is regulated by the EGFR family (43). The expression of MYC was confirmed and it was revealed that the expression of MYC was decreased subsequent to the silencing of PVT1. Therefore, it was concluded that PVT1 activated the EGFR pathway and participated in the progression of ccRCC.

In conclusion, the present study demonstrated that PVT1 expression was significantly upregulated in ccRCC compared with that in normal tissues, and that the high expression of PVT1 was associated with advanced stage and a poor prognosis. Results also indicated that the knockdown of PVT1 induced the apoptosis and cell cycle arrest of ccRCC cells through activation of the EGFR pathway. These findings indicate that PVT1 may serve as a novel therapeutic target in ccRCC.

\section{Acknowledgements}

Not applicable.

\section{Funding}

The present study was supported by the National Natural Science Foundation of China (grant nos. 81672534 and 81472388), the Key Laboratory of Malignant Tumor Molecular Mechanism and Translational Medicine of Guangzhou Bureau of Science and Information Technology [grant no. (2013) 163], the Key Laboratory of Malignant Tumor Gene Regulation and Target Therapy of Guangdong Higher Education Institutes (grant no. KLB09001) and the Guangdong Science and Technology Department (grant no. 2015B050501004).

\section{Availability of data and materials}

All data generated or analyzed during this study are included in this published article.

\section{Authors' contributions}

WX, WL and ZZ designed the experiments and wrote the article. WL and ZZ performed the experiments. HC and YC performed the statistical analysis. All authors have read and approved the final manuscript for publication.

\section{Ethics approval and consent to participate}

All tissue samples were collected with written informed consent from all patients and the study was ethically approved by the Ethic Review Committee of Sun Yat-sen Memorial Hospital.

\section{Consent for publication}

The patients included in the present study gave their consent for the publication of their data.

\section{Competing interests}

The authors declare that they have no competing interests.

\section{References}

1. Siegel RL, Miller KD and Jemal A: Cancer statistics, 2016. CA Cancer J Clin 66: 7-30, 2016.

2. Lipworth L, Tarone RE and McLaughlin JK: The epidemiology of renal cell carcinoma. J Urol 176: 2353-2358, 2006.

3. Paner GP, Stadler WM, Hansel DE, Montironi R, Lin DW and Amin MB: Updates in the eighth edition of the tumor-node-metastasis staging classification for urologic cancers. Eur Urol, Jan 8, 2018 (Epub ahead of print).

4. Dasgupta P, Kulkarni P, Majid S, Varahram S, Hashimoto Y, Bhat NS, Shiina M, Deng G, Saini S, Tabatabai ZL, et al: MicroRNA-203 inhibits long noncoding RNA HOTAIR and regulates tumorigenesis through epithelial-to-mesenchymal transition pathway in renal cell carcinoma. Mol Cancer Ther, Feb 13, 2018 (Epub ahead of print).

5. Hu B, Wang J and Jin X: MicroRNA-138 suppresses cell proliferation and invasion of renal cell carcinoma by directly targeting SOX9. Oncol Lett 14: 7583-7588, 2017.

6. Duns G, van den Berg E, van Duivenbode I, Osinga J, Hollema $\mathrm{H}$, Hofstra $\mathrm{RM}$ and Kok $\mathrm{K}$ : Histone methyltransferase gene SETD2 is a novel tumor suppressor gene in clear cell renal cell carcinoma. Cancer Res 70: 4287-4291, 2010.

7. Kapur P, Peña-Llopis S, Christie A,Zhrebker L,Pavía-Jiménez A, Rathmell WK, Xie XJ and Brugarolas J: Effects on survival of BAP1 and PBRM1 mutations in sporadic clear-cell renal-cell carcinoma: A retrospective analysis with independent validation. Lancet Oncol 14: 159-167, 2013.

8. Gossage L, Murtaza M, Slatter AF, Lichtenstein CP, Warren A, Haynes B, Marass F, Roberts I, Shanahan SJ, Claas A, et al: Clinical and pathological impact of VHL, PBRM1, BAP1, SETD2, KDM6A, and JARID1c in clear cell renal cell carcinoma. Genes Chromosomes Cancer 53: 38-51, 2014.

9. Schmitt AM and Chang HY: Long noncoding RNAs in cancer pathways. Cancer Cell 29: 452-463, 2016.

10. Huarte M: The emerging role of lncRNAs in cancer. Nat Med 21: 1253-1261, 2015.

11. Yang S, Xu J and Zeng X: A six-long non-coding RNA signature predicts prognosis in melanoma patients. Int J Oncol, Feb 7, 2018 (Epub ahead of print).

12. Chen X, Chen Z, Yu S, Nie F, Yan S, Ma P, Chen Q, Wei C, Fu H, $\mathrm{Xu}$ T, et al: Long noncoding RNA LINC01234 functions as a competing endogenous RNA to regulate CBFB expression by sponging miR-204-5p in gastric cancer. Clin Cancer Res, Jan 31, 2018 (Epub ahead of print).

13. Wan L, Sun M, Liu GJ, Wei CC, Zhang EB, Kong R, Xu TP, Huang MD and Wang ZX: Long noncoding RNA PVT1 promotes non-small cell lung cancer cell proliferation through epigenetically regulating LATS2 expression. Mol Cancer Ther 15: 1082-1094, 2016.

14. Sarver AL, Murray CD, Temiz NA, Tseng YY and Bagchi A: MYC and PVT1 synergize to regulate RSPO1 levels in breast cancer. Cell Cycle 15: 881-885, 2016.

15. Zhang XW, Bu P, Liu L, Zhang XZ and Li J: Overexpression of long non-coding RNA PVT1 in gastric cancer cells promotes the development of multidrug resistance. Biochem Biophys Res Commun 462: 227-232, 2015.

16. Cancer Genome Atlas Research Network: Comprehensive molecular characterization of clear cell renal cell carcinoma. Nature 499: 43-49, 2013.

17. Mermel CH, Schumacher SE, Hill B, Meyerson ML, Beroukhim R and Getz G: GISTIC2.0 facilitates sensitive and confident localization of the targets of focal somatic copy-number alteration in human cancers. Genome Biol 12: R41, 2011.

18. Livak KJ and Schmittgen TD: Analysis of relative gene expression data using real-time quantitative PCR and the 2(-Delta Delta C(T)) method. Methods 25: 402-408, 2001.

19. Song W, Dang Q, Xu D, Chen Y, Zhu G, Wu K, Zeng J, Long Q, Wang X, He D and Li L: Kaempferol induces cell cycle arrest and apoptosis in renal cell carcinoma through EGFR/p38 signaling. Oncol Rep 31: 1350-1356, 2014. 
20. Liang L, Li L, Zeng J, Gao Y, Chen YL, Wang ZQ, Wang XY, Chang LS and He D: Inhibitory effect of silibinin on EGFR signal-induced renal cell carcinoma progression via suppression of the EGFR/MMP-9 signaling pathway. Oncol Rep 28: 999-1005, 2012.

21. Clark BS and Blackshaw S: Long non-coding RNA-dependent transcriptional regulation in neuronal development and disease. Front Genet 5: 164, 2014

22. Li P, Ruan X, Yang L, Kiesewetter K, Zhao Y, Luo H, Chen Y, Gucek M, Zhu J and Cao H: A liver-enriched long non-coding RNA, lncLSTR, regulates systemic lipid metabolism in mice. Cell Metab 21: 455-467, 2015.

23. Carrieri C, Forrest AR, Santoro C, Persichetti F, Carninci P, Zucchelli $\mathrm{S}$ and Gustincich S: Expression analysis of the long non-coding RNA antisense to Uchl1 (AS Uchl1) during dopaminergic cells' differentiation in vitro and in neurochemical models of Parkinson's disease. Front Cell Neurosci 9: 114, 2015.

24. Tsoi LC, Iyer MK, Stuart PE, Swindell WR, Gudjonsson JE, Tejasvi T, Sarkar MK, Li B, Ding J, Voorhees JJ, et al: Analysis of long non-coding RNAs highlights tissue-specific expression patterns and epigenetic profiles in normal and psoriatic skin. Genome Biol 16: 24, 2015.

25. Zhang HM, Yang FQ, Chen SJ, Che J and Zheng JH: Upregulation of long non-coding RNA MALAT1 correlates with tumor progression and poor prognosis in clear cell renal cell carcinoma. Tumour Biol 36: 2947-2955, 2015.

26. Hirata H, Hinoda Y, Shahryari V, Deng G, Nakajima K, Tabatabai ZL, Ishii N and Dahiya R: Long noncoding RNA MALAT1 promotes aggressive renal cell carcinoma through Ezh2 and interacts with miR-205. Cancer Res 75: 1322-1331, 2015.

27. Qiao HP, Gao WS, Huo JX and Yang ZS: Long non-coding RNA GAS5 functions as a tumor suppressor in renal cell carcinoma. Asian Pac J Cancer Prev 14: 1077-1082, 2013.

28. Xue S, Li QW, Che JP, Guo Y, Yang FQ and Zheng JH: Decreased expression of long non-coding RNA NBAT-1 is associated with poor prognosis in patients with clear cell renal cell carcinoma. Int J Clin Exp Pathol 8: 3765-3774, 2015.

29. Wu Y, Liu J, Zheng Y, You L, Kuang D and Liu T: Suppressed expression of long non-coding RNA HOTAIR inhibits proliferation and tumourigenicity of renal carcinoma cells. Tumour Biol 35: 11887-11894, 2014.

30. Takahashi Y, Sawada G, Kurashige J, Uchi R, Matsumura T, Ueo H, Takano Y, Eguchi H, Sudo T, Sugimachi K, et al: Amplification of PVT-1 is involved in poor prognosis via apoptosis inhibition in colorectal cancers. Br J Cancer 110: 164-171, 2014.

31. Tseng YY, Moriarity BS, Gong W, Akiyama R, Tiwari A, Kawakami H, Ronning P, Reuland B, Guenther K, Beadnell TC, et al: PVT1 dependence in cancer with MYC copy-number increase. Nature 512: 82-86, 2014.
32. Surendran K, Selassie M, Liapis H, Krigman H and Kopan R: Reduced Notch signaling leads to renal cysts and papillary microadenomas. J Am Soc Nephrol 21: 819-832, 2010.

33. Edge SB and Compton CC: The American Joint Committee on Cancer: The 7th edition of the AJCC cancer staging manual and the future of TNM. Ann Surg Oncol 17: 1471-1474, 2010.

34. Martinou JC and Youle RJ: Mitochondria in apoptosis: Bcl-2 family members and mitochondrial dynamics. Dev Cell 21: 92-101, 2011

35. Kaufmann T, Strasser A and Jost PJ: Fas death receptor signalling: Roles of Bid and XIAP. Cell Death Differ 19: 42-50, 2012.

36. Wei MC, Zong WX, Cheng EH, Lindsten T, Panoutsakopoulou V, Ross AJ, Roth KA, MacGregor GR, Thompson CB and Korsmeyer SJ: Proapoptotic BAX and BAK: A requisite gateway to mitochondrial dysfunction and death. Science 292: 727-730, 2001.

37. Tewari M, Quan LT, O'Rourke K, Desnoyers S, Zeng Z, Beidler DR, Poirier GG, Salvesen GS and Dixit VM: Yama/CPP32 beta, a mammalian homolog of CED-3, is a CrmA-inhibitable protease that cleaves the death substrate poly(ADP-ribose) polymerase. Cell 81: 801-809, 1995.

38. Sherr CJ: Cancer cell cycles. Science 274: 1672-1677, 1996.

39. Chandra A, Lan S, Zhu J, Siclari VA and Qin L: Epidermal growth factor receptor (EGFR) signaling promotes proliferation and survival in osteoprogenitors by increasing early growth response 2 (EGR2) expression. J Biol Chem 288: 20488-20498, 2013.

40. Zhan Y, Chen Y, Liu R, Zhang H and Zhang Y: Potentiation of paclitaxel activity by curcumin in human breast cancer cell by modulating apoptosis and inhibiting EGFR signaling. Arch Pharm Res 37: 1086-1095, 2014.

41. Chen W, Zhong X, Wei Y, Liu Y, Yi Q, Zhang G, He L, Chen F, Liu Y and Luo J: TGF- $\beta$ regulates survivin to affect cell cycle and the expression of EGFR and MMP9 in glioblastoma. Mol Neurobiol 53: 1648-1653, 2016

42. Sigismund S, Avanzato D and Lanzetti L: Emerging functions of the EGFR in cancer. Mol Oncol 12: 3-20, 2018.

43. Chou YT, Lin HH, Lien YC, Wang YH, Hong CF, Kao YR, Lin SC, Chang YC, Lin SY, Chen SJ, et al: EGFR promotes lung tumorigenesis by activating miR-7 through a Ras/ERK/Myc pathway that targets the Ets2 transcriptional repressor ERF. Cancer Res 70: 8822-8831, 2010.

This work is licensed under a Creative Commons Attribution-NonCommercial-NoDerivatives 4.0 International (CC BY-NC-ND 4.0) License. 